\title{
EFEITO PROTETOR DO ENRIQUECIMENTO AMBIENTAL NA MANIFESTAÇÃO TARDIA DA ANSIEDADE E DO DÉFICIT DE EXTINÇÃO DA MEMÓRIA DE MEDO INDUZIDOS POR ESTRESSE AGUDO EM RATOS: O PAPEL DA SINALIZAÇÃO DE GLICOCORTICOIDE NO COMPLEXO BASOLATERAL DA AMÍGDALA
}

Tese apresentada ao programa de Pós-Graduação em Farmacologia do Instituto de Ciências Biomédicas da Universidade de São Paulo para obtenção do Título de Doutor em Ciências

Área de concentração: Farmacologia

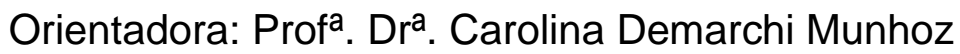

Versão original 


\section{RESUMO}

Novaes LS. Efeito protetor do enriquecimento ambiental na manifestação tardia da ansiedade e do déficit de extinção da memória de medo induzidos pelo estresse agudo em ratos: o papela da sinalização de glicocorticoide no complexo basolateral da amígdala. [Tese (Doutorado em farmacologia)]. São Paulo: Instituto de Ciências Biomédicas, Universidade de São Paulo, São Paulo; 2017.

As consequências de eventos estressantes sobre a saúde humana, principalmente relacionada à condição psiquiátrica, tem ganhado notoriedade nos últimos anos em decorrência do crescente número de comorbidades associadas ao estresse registradas nas grandes cidades. Transtornos emocionais relacionados a sintomas de ansiedade são comuns dentre os relatados na clínica psiquiátrica e ganham importância em trabalhos científicos devotados ao estresse, que lançam mão de abordagens diversas para compreender os mecanismos neurobiológicos subjacentes à a persistência de sintomas ansiosos decorrentes de um evento estressante. Trabalhos prévios mostraram que tanto o estresse agudo de contenção quanto a administração sistêmica de corticosterona (CORT, hormônio glicocorticoide murino) promovem, 10 dias depois, comportamento do tipo ansioso e remodelamento dendrítico no complexo basolateral da amígdala (BLA) em ratos. Além disso, alguns trabalhos recentes mostraram que a exposição ao enriquecimento ambiental (EA) reverteu o efeito ansiogênico e sobre a modulação dendrítica do BLA induzidos por estresse repetido. Em trabalho recente, nós verificamos que o EA preveniu o efeito ansiogênico imediato do estresse agudo de contenção. Esse efeito protetor do EA pareceu estar relacionado a seu efeito preventivo no aumento da atividade neuronal e do receptor de glicocorticoide (GR) no BLA. No presente trabalho, nós verificamos que o EA preveniu tanto o surgimento do comportamento do tipo ansioso quanto o déficit de extinção da memória de medo induzidos por estresse agudo de contenção verificados 10 dias depois. Porém, não está claro se esses efeitos estão relacionados com alterações na árvore dendrítica do BLA e/ou com a atividade de GR no mesmo núcleo. Dessa forma, um dos objetivos centrais do trabalho foi determinar se o efeito preventivo do EA na persistência das alterações comportamentais, e nas alterações morfológicos no BLA, induzidas por estresse agudo, são atribuidas às ações de CORT. Nós observamos que o efeito preventivo do EA na ansiedade e no déficit de extinção, verificados 10 dias após o estresse, não estão relacionados à prevenção no aumento da densidade de espinhos dendríticos do BLA. Além disso, ainibição da síntese de CORT por metirapona preveniu a emergência do comportamento do tipo ansioso 10 dias após o estresse, indicando que a sinalização desse hormônio é crucial para os efeitos comportamentais tardios relacionados ao estresse agudo. Finalmente, lançando mão do uso da tecnologia do DNA recombinante, nós verificamos que a inibição da atividade genômicoa de GR no BLA preveniu o comportamento do tipo ansioso manifestado 10 dias após o estresse

Palavras-chave: Estresse. Enriquecimento ambiental. Ansiedade. Extinção da memória de medo. Amígdala. 


\begin{abstract}
Novaes LS. Environmental enrichment protection on acute stress-induced late anxiety-like behavior and fear extinction impairment in rats: role of glucocorticoid receptor signaling in the basolateral nucleus of amygdala. [PhD Thesis (Pharmacology)], São Paulo: Instituto de Ciências Biomédicas, Universidade de São Paulo; 2013.
\end{abstract}

The consequences of stressful events on human health, especially related to psychiatric disorders, have gained attention in recent years due the increasing number of comorbidities associated to stress in large cities. Anxiety-related disorders are common among the psychiatric patients and are widely present in studies devoted to stress, which use different approaches to investigate the neurobiological mechanisms underlying the persistence of anxiety symptoms caused by a stressful event. Either acute restraint stress or systemic injection of corticosterone (CORT, a rodent glucocorticoid) in rats leads to enhanced anxiety-like behavior and dendritic branch remodeling in the basolateral amygdala complex (BLA) 10 days later. Also, some studies showed that exposure to environmental enrichment (EE) reverted the longlasting anxiety-like behavior and the repeated stress-induced BLA dendritic hypertrophy in rats. In a recent study, we found that EE prevented anxiety-related behavior in adult rats observed immediately after acute restraint stress. This protective role of EE appears to be due to the prevention of the stress-induced increase in neuronal activity and in glucocorticoid receptor (GR) nuclear activity in the BLA. In this study, we showed that EE prevented the restraint stress-induced long-lasting anxiety and contextual fear extinction impairment in adult rats. However, it is not yet clear if this protective role of EE is related to changes in the dendritic branch and/or in the GR signaling in the BLA. The present study sought to determine whether the preventive effect of EE on persistent stress-related changes in behavior as well as in the BLA morphology and activity are attributed to CORT signaling. We found that the EEinduced protection on anxiety-like behavior and fear extinction impairment 10 days after acute restraint stress was not related to prevention of the increase in spine density in BLA. Moreover, systemic injection of GC synthesis inhibitor (metyrapone) prevented the anxiety-like behavior 10 days after stress, showing that GC signaling during stress is crucial to late stress-related behavior. Finally, by antagonizing the genomic signaling of the endogenous GR in the BLA, through the use of recombinant DNA technology, we prevented the emergence of anxiety-related behavior 10 days after acute stress.

Keywords: Stress. Environmental enrichment. Anxiety. Fear extinction. Amygdala. 


\section{INTRODUÇÃO}

As respostas fisiológica e comportamental do organismo a estímulos estressores possuem um importante valor adaptativo, na medida em que o preparam para lidar com uma situação de risco. Porém, a recorrente submissão a estímulos dessa ordem implica em alterações adaptativas em sistemas endócrinos e de mediadores neurais, como os hormônios glicocorticoides (GCs) e as catecolaminas (Mclntyre et al., 2012). Esses mediadores estão diretamente implicados no desenvolvimento de transtornos psiquiátricos de humor e ansiedade, como depressão, síndrome do pânico, transtorno obsessivo compulsivo e transtorno do estresse pós-traumático (TEPT; Abelson et al., 2007; Kluge et al., 2007; Ressler e Nemeroff, 1999). Dados divulgados pela Secretaria de Previdência do Ministério da Fazenda em parceria com o Instituto Nacional do Seguro Social (INSS) ${ }^{1}$ mostram que, entre os anos de 2014 e 2016, foram concedidos no Brasil 538.493 auxílios acidentários na categoria transtornos mentais e comportamentais (90\% referiam-se a problemas como estresse, episódios depressivos, alteração de humor e ansiedade), equivalendo a cerca de $9,3 \%$ do total de auxílios concedidos, sendo a terceira causa mais recorrente de afastamento no período. Levantamento realizado por Andrade et al. (2012) apontou que cerca de 30\% da população residente na região metropolitana de São Paulo apresenta algum tipo de transtorno mental, sendo os mais comuns aqueles agrupados nas categorias de ansiedade (19\%) e distúrbios de humor (11\%).

A recordação de um evento ameaçador, tanto quanto a resposta fisiológica e comportamental imediata a um dado estímulo estressor, também possui um valor biológico adaptativo importante, na medida em que prepara o organismo para gerar uma resposta mais eficaz a uma eventual situação de risco futura. Porém, a recordação persistente de um evento estressante agudo pode desencadear uma condição patológica associada à ansiedade, onde a memória recordada assume uma característica traumática recorrentemente evocada, condição característica de pacientes com TEPT (American Psychiatric Association: Diagnostic and statistical manual of mental disorders: DSM-V, 2013).

\footnotetext{
1 Anuário Estatístico da Previdência Social/Ministério da Fazenda, Secretaria da Previdência, Empresa de Tecnologia e Informações da Previdência - Ano 9 (2014-2016); v.23, p. 1-934.
} 
A esse respeito, muitos pesquisadores estão investigando quais condições levam alguns indivíduos a desenvolverem TEPT, lançando mão de abordagens que variam desde a caracterização genética idiossincrática do indivíduo até a influência de fatores de riscos ambientais e, mais recentemente, a combinação de ambos os fatores através da investigação de mecanismos epigenéticos da modulação genômica (ver McEwen et al., 2012). Nesse contexto, diversos trabalhos relatam a influência modulatória que o estado de excitação emocional (do termo inglês arousal), durante a exposição a um evento, exerce sobre a memória, atribuindo uma valência emocional ao fato a ser recordado e, assim, modificando sua persistência em comparação a fatos extraídos de contextos emocionalmente neutros (para um a ampla revisão, ver de Quervainet al., 2017; McEwen et al., 2012; McGaugh, 2004).

O condicionamento clássico de medo é um dos paradigmas mais usados em animais de experimentação para o estudo da memória emocional e é extensamente utilizado como modelo de estudos de desordens associadas ao estresse e ansiedade (Fendt e Fanselow, 1999; LeDoux, 2000). Nesse paradigma, durante o período de treino, o rato ou camundongo é exposto a um estímulo condicionado neutro (EC; por exemplo, um sinal luminoso, sonoro ou olfativo) pareado a um estímulo incondicionado (El) aversivo, por exemplo, um choque nas patas ou um ruído estridente. No dia do teste, quando se verifica a memória adquirida no treino, a apresentação apenas do EC desperta uma resposta emocional de medo, mesmo na ausência do El, tal como congelamento (freezing), aumento do reflexo de sobressalto e supressão de comportamento exploratório. Além disso, há manifestações autonômicas e neuroendócrinas como o aumento da liberação de hormônios do eixo hipotálâmico-hipofisário-adrenal (HPA), entre eles o hormônio adrenocorticotrófico (ACTH) e a corticosterona (CORT, principal GC murino, análoga ao cortisol humano; Fendt e Fanselow, 1999). Essas respostas condicionadas podem ser observadas quando o animal é submetido à arena de testes onde recebeu o estímulo aversivo (EI), mesmo na ausência do EC. Nesse caso, o condicionamento está associado ao contexto.

A amígdala figura como estrutura chave no condicionamento aversivo clássico (ver LeDoux, 2000). Estudos anatômicos, eletrofisiológicos e comportamentais sugerem que a convergência dos estímulos condicionado e incondicionado (de ordem sensorial) ocorre no complexo basolateral da amígdala (BLA - que inclui os núcleos lateral, basolateral e basomedial), cujas projeções determinam direta, ou 
indiretamente, a resposta comportamental do animal (Amaral et al., 1992; Fendt and Fanselow, 1999; Gallagher and Holland, 1994; Janak and Tye, 2015; McDonald, 1998; Sah et al., 2003; Salzman and Fusi, 2010; Sarter and Markowitsch, 1985). Lesões ou inibição dos neurônios da região prejudica o condicionamento de medo (Amano et al.,2011; Anglada-Figueroa e Quirk, 2005; Hatfield et al., 1996; Maren et al., 2001), ao passo que a estimulação de populações neuronais do BLA favorece o pareamento com um estímulo neutro para, posteriormente, desencadear uma resposta de medo (Johansen et al., 2010; Yiu et al., 2014). A apresentação apenas do EC, em animais que tiveram o estímulo pareado com o El, evoca respostas eletrofisiológicas mais acentuadas nos neurônios do núcleo lateral da amígdala quando comparados a animais que não tiveram o dado estímulo pareado com o El (Collins e Paré, 2000; Quirk et al., 1995). Em trabalho recente, Gore e colegas (2015) mostraram que os neurônios do BLA ativados durante a apresentação do El, quando estimulados fora do contexto em que este foi apresentado, gera a mesma resposta comportamental desencadeada pelo El. Além disso, os mesmos neurônios ativados durante a apresentação do El são necessários para a expressão do condicionamento quando da apresentação do EC, se este foi pareado com o El.

Nas últimas décadas, diversos achados trouxeram avanços importantes sobre o substrato neural subjacente ao processo mnemônico associado a experiências estressantes, visando compreender como o estado de excitação emocional, além do componente potencialmente aversivo intrínseco ao próprio evento, modula o processamento da memória (de Quervain et al., 2017; Joëls et al., 2006; de Kloet et al., 1999; McEwen e Sapolsky, 1995; McEwenn et al., 2015; Zoladz et al., 2011). Dentro dessa perspectiva, muitas abordagens investigam a influência dos mediadores neuroendócrinos do estresse nas diferentes fases da formação ou expressão da memória. GCs, liberados pela glândula suprarrenal em decorrência de eventos estressantes, ganham destaque nesses trabalhos, fundamentalmente por estarem implicados em diversas patologias associadas ao estresse (de Kloet et al., 2005; McEwenn et al., 2015; de Quervain et al., 2017). De forma geral, sabe-se que a elevação sistêmica de CORT em roedores resulta em aumento na capacidade de consolidação da memória de informações novas, porém prejudica o processo de recordação de memórias anteriormente armazenadas (de Quervain et al., 1998; Roozendaal et al., 1999). Por outro lado, a elevação crônica dos níveis do mesmo hormônio está associada a prejuízos no desempenho em testes de cognição 
(McEwen, 2001, Sapolsky , 2000). Estudos utilizando modelos de condicionamento clássico mostraram que a administração sistêmica de CORT resulta em aumento de consolidação de memória quando efetuada antes, ou imediatamente após, o treino de aquisição da memória (Buchanan e Lovallo, 2001; Cordero et al., 2002; Roozendaal et al., 1999). Um aspecto muito importante a ser destacado a respeito do papel dos GCs sobre a memória é sua atuação potencializadora apenas quando há um componente aversivo, ou de alta excitação emocional, intrínsecos ao teste. Nesse sentido, em modelos experimentais nos quais não há associação da aversividade com o fato ou evento a ser recordado, o aumento sistêmico de CORT não exerce qualquer influência no processo de aquisição de memória (Buchanan e Lovallo, 2001; Kuhlmann e Wolf, 2006; Okuda et al., 2004).

A influência de estímulos estressores exógenos ao contexto do paradigma de aprendizado sobre a memória emocional também depende do processamento das informações pela amígdala (Davis, 1992; Liu et al., 1999; Pelletier et al., 2005). A execução de atividades estressantes, ou de alta excitação emocional, que elevam, portanto, a liberação de hormônios glicocorticoides pela glândula adrenal, também ativam a amígdala (Pelletier et al., 2005). Além de serem hormônios lipofílicos e, assim, atingirem rapidamente uma extensa área do organismo, incluindo o encéfalo, a amígdala contém consideráveis concentrações de receptores de mineralocorticoides (MR) e de glicocorticoides (GR) (Patel et al., 2000; Pryce, 2008). Kvushansky e Richter-Levin (2006) mostraram uma relação diretamente proporcional entre o aumento da concentração sérica de CORT e a atividade dos neurônios da amígdala. Dados obtidos em humanos e em animais apontam o papel central da amígdala em uma variedade de processos que envolvem informações emocionalmente excitáveis, incluindo influências sobre a atenção, percepção, aprendizado e memória (LeDoux, 2003; Phelps e LeDoux, 2005). O BLA, nesse contexto, ocupa um papel chave. Lesões nessa estrutura, por exemplo, bloqueiam os efeitos sobre a modulação da memória induzida por administração sistêmica pós-treino de CORT (McGaugh, 2004; Roozendaal e McGaugh, 1996), efeito que independe do núcleo central da amígdala (CeA). Além disso, infusões pós-treino de CORT no BLA, mas não no CeA, promovem aumento de consolidação de memória (Roozendaal e McGaugh, 1997). Já a infusão de antagonista de GR (RU-486) nessa estrutura prejudica, ou até bloqueia, o efeito potencializador decorrente da exposição ao estresse no processo de consolidação da memória (Conrad e MacMillan, 2004; Perusini et al., 2016). 
Trabalhos desenvolvidos com humanos complementam achados obtidos com o uso de animais, mostrando grandes avanços na compreensão do papel da amígdala na atribuição de valência emocional ao estímulo apreendido. Em testes de condicionamento clássico, pacientes com transtornos de ansiedade apresentam maior aquisição e retenção de um estímulo novo associado a uma situação de medo do que indivíduos controles. Nesses trabalhos, a atividade da amígdala novamente se destaca. No condicionamento de medo, por exemplo, trabalhos com ressonância magnética funcional mostraram um aumento significativo da atividade da amígdala durante a fase de aquisição em pacientes ansiosos (Büchel et al., 1998; LaBar et al., 1998). Outros estudos mostraram que pacientes ansiosos tendem a apresentar atenção seletiva a eventos de cunho emocional negativo (ou aversivo) ou, até, a interpretar estímulos de caráter dúbio (sem clara distinção entre aversão e neutralidade) como sendo de ordem aversiva, efeitos acompanhados pelo aumento de atividade da amígdala (Bishop et al., 2004; Pessoa et al., 2002; Vuilleumier et al., 2001). Em conjunto, esses dados apontam que a ansiedade, ou o estado de excitação emocional elevada, promove aumento da aquisição de memória associada a componentes aversivos, quadro que pode ser dependente de um processo de atenção seletiva a estímulos supostamente ameaçadores, além de predispor o sujeito a interpretar estímulos, aparentemente neutros, como aversivos (Bishop, 2007).

A extinção da memória de medo, um paradígma experimental que consiste na redução da resposta apreensiva mediante apresentações sucessivas do EC, possui um correlato importante com a terapia cognitivo-comportamental (Milad e Quirk, 2012; Pitman et al, 2012; Singewald et al, 2015; Yehuda e LeDoux, 2007). Trata-se de um fenômeno baseado na ausência de reforço da resposta apreendida mediante a apresentação do EC. Nesse sentido, a extinção da memória apreendida é um processo ativo de aprendizado, distinto do processo de aprendizado que levou à resposta de medo (Berman e Dudai, 2001; Myers e Davis, 2002).

A dependência de informações contextuais para que a extinção ocorra de forma eficiente traz implicações de relevância clínica importantes, visto ser um dos maiores desafios da psicoterapia chegar a uma redução da resposta apreensiva ou de medo que transcenda o ambiente terapêutico (ver Maren et al., 2013). O BLA, além de um papel crucial na aquisição e expressão do medo apreendido (LeDoux, 2000), também possui um papel central no processo de extinção da memória em modelos animais (Maren e Quirk, 2004). A inibição de síntese proteica ou a infusão de antagonista do 
receptor subtipo NMDA para o glutamato diretamente no BLA prejudica a consolidação da extinção do medo condicionado (Falls et al., 1992; Lee e Kim, 1998), enquanto que a infusão de agonista do receptor NMDA no mesmo núcleo facilita a extinção (Ledgerwood et al., 2003; Walker et al., 2002). Do ponto de vista celular, uma questão intrigante é compreender como o processamento do estímulo condicionado recruta neurônios do BLA para manifestar a resposta de medo e, a depender de um processo de plasticidade (Herry et al., 2010; Quirk e Muller, 2008) decorrente da reexposição ao mesmo estímulo condicionado, porém na ausência do estímulo incondicionado, a resposta é suprimida. Sabe-se que em roedores, populações neuronais específicas do BLA apresentam aumento dos disparos após o condicionamento de medo e essas mesmas populações reduzem a magnitude desses disparos após o processo de extinção (Repa et al., 2001). Paralelamente, algumas populações codificam o processo de extinção, aumentando a atividade de seus neurônios em decorrência da exposição ao treino de extinção (Hobin et al., 2003).

Além das implicações do estresse e da sinalização mediada por CORT na aquisição e consolidação da memória de medo (de Quervain et al., 2017; McGaugh e Roozendall, 2002), recentes evidências vêm mostrando seus efeitos sobre a aquisição e consolidação da extinção da memória. A exposição ao estresse de plataforma suspensa 30 minutos antes do treino de extinção prejudicou a extinção da memória de medo ao contexto (Xu et al., 1997; Xu et al., 1998), ao passo que estresse único prolongado ( $2 \mathrm{~h}$ de estresse de contenção, 20 min de nado forçado e perda de consciência por inalação de éter) prejudicou a extinção de medo ao contexto dos animais quando foram testados 7 dias após o estresse (Knox et al., 2012a; Knox et al., 2012b; Yamamoto et al., 2008). Por outro lado, e de forma contra intuitiva, a inibição da liberação sistêmica de CORT antes do treino de extinção no paradigma pavloviano clássico prejudicou a retenção da extinção da memória (Barrett e Gonzalez-Lima, 2004). No teste de sobressalto potencializado pelo medo, as sucessivas sessões de extinção aumentaram as concentrações séricas do hormônio, ao passo que a administração do glicocorticoide sintético dexametasona facilitou a extinção quando infundida antes, ou imediatamente após, as sessões de extinção (Yang et al., 2006). A infusão intra BLA de antagonista de GR (RU-486) preveniu o efeito facilitatório da administração periférica de Dexametasona (glicocorticoide sintético) na extinção da memória, enquanto que a infusão de agonista de GR (RU28362) intra BLA promoveu aumento de extinção (Yang et al., 2006), sugerindo que o 
BLA é uma região crítica na modulação da memória exercida pelas alterações nas concentrações de CORT. É interessante destacar que pacientes com TEPT, além de apresentarem déficit de extinção de memória traumática (Rothbaun e Davis, 2003), também apresentam alterações nas concentrações de glicocorticoides no plasma e urina, e feedback negativo aumentado do eixo HPA (Yehuda, 2001). Outros trabalhos mostraram, ainda, que a administração diária de baixas doses de hidrocortisona (um outro glicocorticoide sintético) reduz os sintomas clínicos em pacientes com TEPT (Aerni et al., 2004; Soravia et al., 2006).

Além de possuir um papel chave nos efeitos do estresse sobre os diferentes processos de memória emocional, diversos trabalhos vêm mostrando que o BLA também protagoniza o efeito ansiogênico do estresse não relacionado à memória (Davis, 1992; Gross and Hen, 2004; LeDoux, 2000; Rosen and Schulkin, 1998; Rodrigues et al., 2009). Alguns trabalhos mostraram que o estresse crônico de contenção em ratos ( $2 \mathrm{~h}$ por 10 dias consecutivos) promove alterações na morfologia da árvore dendrítica do BLA, como o aumento do comprimento de cada segmento e do número de bifurcações (Mitra et al., 2005; Vyas et al., 2002), sugerindo um aumento da hiperatividade dos neurônios dessa região. Outros trabalhos mostraram que o estresse agudo de contenção (2h), assim como a administração sistêmica de uma única dose de CORT, geraram o mesmo efeito comportamental que o estresse crônico (Kim et al., 2014; Mitra et al., 2005; Mitra e Sapolsky, 2008; Rao et al., 2012). Interessantemente, nesses trabalhos, o comportamento ansioso dos animais foi verificado 10-12 dias após a infusão de CORT ou o estímulo estressor e coincidiu com o período em que também foram verificadas alterações na árvore dendrítica do BLA (nesse caso, aumento da densidade dos espinhos dendríticos), sugerindo a necessidade de um período de latência para que sejam verificados os efeitos morfológicos do estresse no BLA. Em conjunto, esses trabalhos sugerem que há uma correlação entre os efeitos do estresse sobre a manifestação do comportamento ansioso e seu efeito sobre a árvore dendrítica do BLA, além de indicarem que os efeitos do estresse sejam mediados pela CORT.

Abordagens distintas vêm sendo utilizadas para verificar fatores predisponentes ou protetores do organismo à submissão a estímulos estressores, dentre elas as condições ambientais às quais os mesmos são submetidos. $O$ enriquecimento ambiental (EA) é um modelo experimental amplamente utilizado para esse fim, com conhecidas propriedades ansiolíticas (Benaroya-Milshtein et al., 2004; 
Friske e Gammie, 2005; Huzard et al., 2015; Koe et al., 2016; Pietropaolo et al., 2014; Revenelle et al., 2014; Roy et al., 2001) e tem sido amplamente utilizado como modelo de indução de proteção ao organismo frente a estímulos estressores em diferentes modelos animais (ver Fox et al., 2006). Trata-se de um modelo experimental no qual os animais são acondicionados em caixas moradia que propiciam o aumento de estímulos sensórios, motores e sociais em relação às condições padrões oferecidas a animais de experimentação. De modo geral, o EA consiste no acondicionamento de grupos de animais em caixas relativamente grandes nas quais são introduzidos periodicamente objetos e brinquedos, de tal forma a criar um ambiente que potencialize as interações sociais e estimule o comportamento exploratório e motor (Mohammed et al., 2002; van Praag et al., 2000).

Embora muitos trabalhos tenham mostrado o efeito protetor do EA em diferentes transtornos induzidos por estresse, ainda não está claro como esse tipo de moradia exerce seus efeitos biológicos. Em trabalho recente (Novaes et al., 2017), obtivemos alguns resultados que nos ajudam a esclarecer os mecanismos neurobiológicos subjacentes aos efeitos protetores do EA sobre o comportamento do tipo ansioso induzido pelo estresse agudo. De forma geral, verificamos que animais acomodados em gaiolas enriquecidas são resilientes ao desencadeamento de comportamento do tipo ansioso induzido por estresse agudo de contenção e apresentaram menor expressão de EGR-1 no BLA, um marcador indireto de atividade neuronal. Contra intuitivamente, o EA não preveniu o aumento das concentrações séricas de CORT induzido pelo estresse, entretanto, nós observamos aumento da atividade nuclear de GR apenas nos animais não enriquecidos. Esses dados são interessantes na media em que muitos dos trabalhos relacionam os efeitos protetores do EA a seu efeito modulatório sobre o eixo HPA (Belz et al., 2003; Francis et al., 2002; Morley-Fletcher et al., 2003; Moncek et al., 2004; Roy et al., 2001; Welberg et al., 2006).

Pertencente à família dos receptores nucleares, o GR encontra-se no citoplasma da célula acoplado a algumas proteínas acessórias (chaperonas, como a Hsp90; co-chaperonas, como a Fkbp5; co-reguladores, como a CBP) e, quando ligado ao hormônio glicocorticoide, dissocia-se dessas proteínas e migra, em dímeros ou não, para o núcleo celular (ver Duque e Mnhoz, 2016). No núcleo celular, o complexo 
GR-ligante atua como fator de transcrição através da transativação ou transrepressão de genes, ligando-se com alta afinidade aos elementos responsivos a glicocorticoides (GRE), localizados na região promotora ou no zona intra-gênica dos genes alvos (Kadmiel e Cidlowisk, 2014; Ratman et al., 2013). Dados da literatura mostram que o aumento da atividade nuclear de GR, desencadeado por estresse, é um fenômeno diretamente relacionado ao aumento da resposta eletrofisiológica dos neurônios do BLA (Duvarci e Paré, 2007), fenômeno atrelado à gênese da resposta do tipo ansiosa (Drevets et al., 1992). Ademais, evidências apontam que esse efeito modulatório de GR sobre a excitabilidade dos neurônios do BLA se deve à ação genômica do receptor (Duvarci e Paré, 2007).

\section{CONCLUSÃO}

De forma geral, nossos resultados mostram que existe um claro papel protetor do EA contra os efeitos comportamentais que o estresse agudo promove tardiamente nos ratos, porém muitos marcadores biológicos aos quais são atribuidos esses efeitos comportamentais do estresse não necessariamente correspondem aos os mecanismos através dos quais o EA exerce seu efeito protetor. Tratando-se de um protocolo experimental no qual os animais são submetidos a uma ampla variedade de estímulos, por um período relativamente longo (mais longo que o período de latência entre o estresse e a verificação de seus efeitos), o EA provavelmente promove uma plasticidade no sistema nervoso central (e talvez periférico) compensatória aos efeitos do estresse, não necessariamente na direção contrária a esses efeitos. Um exemplo de ação compensatória do EA poderia ser, por exemplo, a modulação sobre a atividade genômica de GR no BLA que, de alguma forma, compensaria os efeitos periféricos da ação do estresse sobre o eixo HPA. Uma evidencia prévia de que o EA poderia exercer seu efeito protetor frente ao estresse mediante o controle da atividade genômica de GR nos levou a investigar, e concluir, que, de fato, a ação desse receptor no BLA está diretamente relacionada aos efeitos ansiogênicos do estresse, a despeito de um eventual efeito sobre o exio HPA e a morfologia dendrítica do BLA. Embora não possamos concluir a respeito do papel da ação de GR no BLA sobre os efeitos do estresse na memória (mas também não podemos excluir essa possibilidade), esse achado nos aponta para um efeito mais localizado (intracelular) do estresse sobre uma determinada estrutura encefálica, o que poderia, inclusive, nos ajudar enteder como 
alterações em subpopulações específicas de neurônios no sistema nervoso central influenciam o comportamento do animal. 


\section{REFERÊNCIAS ${ }^{2}$}

Abelson JL, Khan S, Liberzon I, Young EA. HPA axis activity in patients with panic disorder: review and synthesis of four studies. Depress Anxiety. 2007; 24(1):66-76.

Adamec RE, Burton P, Shallow T, Budgell J. Unilateral block of NMDA receptors in the amygdala prevents predator stress-induced lasting increases in anxiety-like behavior and unconditioned startle--effective hemisphere depends on the behavior. Physiology \& behavior. 1999; 65:739-751.

Aerni A, Traber R, Hock C, Roozendaal B, Schelling G, Papassotiropoulos A, Nitsch RM, Schnyder U, de Quervain DJ. Low-dose cortisol for symptoms of posttraumatic stress disorder. Am J Psychiatry. 2004; 161(8):1488-90.

Akirav I, Maroun M. The role of the medial prefrontal cortexamygdala circuit in stress effects on the extinction of fear. Neural Plast. 2007.

Akirav I, Raizel H, Maroun M. Enhancement of conditioned fear extinction by infusion of the $\operatorname{GABA}(A)$ agonist muscimol into the rat prefrontal cortex and amygdala. Eur J Neurosci. 2006; 23(3):758-64.

Amano T, Duvarci S, Popa D, Paré D. The fear circuit revisited: contributions of the basal amygdala nuclei to conditioned fear. J Neurosci. 2011; 31(43):15481-9.

Amaral D, Price J, Pitkanen A, Carmichael S. The Amygdala: Neurobiological Aspects of emotion, Memory, and Mental Dysfunction. J. Agleton, ed. 1992; New York, pp 116.

Ambroggi F, Ishikawa A, Fields HL, Nicola SM. Basolateral amygdala neurons facilitate reward-seeking behavior by exciting nucleus accumbens neurons. Neuron. 2008; 59(4):648-61.

American Psychiatric Association: Diagnostic and statistical manual of mental disorders: DSM-IV-TR. 5ed. Arlington, VA: American Psychiatric Publishing. 2013.

Andrade LH, Wang YP, Andreoni S, Silveira CM, Alexandrino-Silva C, Siu ER, Nishimura R, Anthony JC, Gattaz WF, Kessler RC, Viana MC. Mental disorders in

\footnotetext{
2 De acordo com:

International Committee of Medical Journal Editors. [Internet]. Uniforms requirements for manuscripts submitted to Biomedical Journal: sample references. [updated $2011 \mathrm{Jul} 15$ ]. Available from:

http://www.icmje.org
} 
megacities: findings from the São Paulo megacity mental health survey, Brazil. PLoS One. 2012; 7(2):e31879.

Anglada-Figueroa D, Quirk GJ. Lesions of the basal amygdala block expression of conditioned fear but not extinction. J Neurosci. 2005; 25(42):9680-5.

Armario A, Escorihuela RM, Nadal R. Long-term neuroendocrine and behavioural effects of a single exposure to stress in adult animals. Neurosci Biobehav Rev. 2008; 32(6):1121-35.

Ashokan A, Hegde A, Mitra R. Short-term environmental enrichment is sufficient to counter stress-induced anxiety and associated structural and molecular plasticity in basolateral amygdala. Psychoneuroendocrinology. 2016; 69, 189-196.

Baldi E, Bucherelli C. Brain sites involved in fear memory reconsolidation and extinction of rodents. Neurosci Biobehav Rev. 2015; 53:160-90.

Barrett D, Gonzalez-Lima F. Behavioral effects of metyrapone on Pavlovian extinction. Neurosci Lett. 2004; 371(2-3):91-6.

Belda X, Fuentes S, Nadal R, Armario A. A single exposure to immobilization causes long-lasting pituitary-adrenal and behavioral sensitization to mild stressors. Horm Behav. 2008; 54(5):654-61.

Belda X, Márquez C, Armario A. Long-term effects of a single exposure to stress in adult rats on behavior and hypothalamic-pituitary-adrenal responsiveness: comparison of two outbred rat strains. Behav Brain Res. 2004;154(2):399-408.

Belova MA, Paton JJ, Morrison SE, and Salzman CD. Expectation modulates neural responses to pleasant and aversive stimuli in primateamygdala. Neuron. 2007; 55, 970-984.

Belz EE, Kennell JS, Czambel RK, Rubin RT, Rhodes ME. Environmental enrichment lowers stress-responsive hormones in singly housed male and female rats. Pharmacol Biochem Behav. 2003; 76(3-4):481-6.

Benaroya-Milshtein N, Hollander N, Apter A, Kululansky T, Raz N. et al. Environmental enrichment in mice decreases anxiety, attenuates stress responses and enhances natural killer cell activity. European Journal of Neuroscience. 2004; 20:1341-1347. 
Berlau DJ, McGaugh JL.. Enhancement of extinction memory consolidation: the role of the noradrenergic and GABAergic systems within the basolateral amygdala. Neurobiol. Learn. Mem. 2006; 86:123-132.

Berman DE, Dudai Y. Memory extinction, learning anew, and learning the new: dissociations in the molecular machinery of learning in cortex. Science. 2001; 291(5512):2417-9.

Bermudez MA, Schultz W. Reward magnitude coding in primate amygdala neurons. J. Neurophysiol. 2010; 104, 3424-3432.

Bignante EA, Paglini G, Molina VA. Previous stress exposure enhances both anxietylike behaviour and p35 levels in the basolateral amygdala complex: modulation by midazolam. European neuropsychopharmacology: the journal of the European College of Neuropsychopharmacology. 2010; 20:388-397.

Bishop SJ, Duncan J, Lawrence AD. State anxiety modulation of the amygdala response to unattended threat-related stimuli. J Neurosci. 2004; 24(6):10364-8.

Bishop SJ. Neurocognitive mechanisms of anxiety: an integrative account. Trends Cogn Sci. 2007; 11(7):307-16.

Bosscher KD. How glucocorticoid receptors modulate the activity of other transcription factors: A scope beyond tethering. Mol Cell Endocrinol. 2013; 5;380(1-2):41-54.

Brown SM, Henning S, Wellman CL. Mild, short-term stress alters dendritic morphology in rat medial prefrontal cortex. Cereb Cortex. 2005; 15(11):1714-22.

Bryant, RA, Felmingham, K., Kemp, AH, Barton, M, Peduto, AS, Rennie, C, Gordon, E, Williams, LM, 2005. Neural networks of information processing in posttraumatic stress disorder: a functional magnetic resonance imaging study. Biol. Psychiatry. 2005; 58, 111-118.

Buchanan TW, Lovallo WR. Enhanced memory for emotional material following stresslevel cortisol treatment in humans. Psychoneuroendocrinology. 2001; 26(3):307-17.

Büchel C, Morris J, Dolan RJ, Friston KJ. Brain systems mediating aversive conditioning: an event-related fMRI study. Neuron. 1998; 20(5):947-57.

Camp MC, Macpherson KP, Lederle L, Graybeal C, Gaburro S, Debrouse LM et al. Genetic strain differences in learned fear inhibition associated with variation in 
neuroendocrine, autonomic, and amygdala dendritic phenotypes. Neuropsychopharmacology. 2012; 37: 1534-1547.

Cardinal RN, Parkinson JA, Hall J, Everitt BJ. Emotion and motivation: the role of the amygdala, ventral striatum, and prefrontal cortex. Neurosci Biobehav Rev. 2002; 26(3):321-52.

Charney DS, Deutch AY, Krystal JH, Southwick SM, Davis M. Psychobiologic mechanisms of posttraumatic stress disorder. Arch Gen Psychiatry. 1993; 50:294-305.

Collins DR, Paré D. Differential fear conditioning induces reciprocal changes in the sensory responses of lateral amygdala neurons to the CS(+) and CS(-). Learn Mem. 2000; 7:97-103.

Conrad CD, MacMillan DD 2nd, Tsekhanov S, Wright RL, Baran SE, Fuchs RA. Influence of chronic corticosterone and glucocorticoid receptor antagonism in the amygdala on fear conditioning. Neurobiol Learn Mem. 2004; 81(3):185-99.

Cordero MI, Kruyt ND, Merino JJ, Sandi C. Glucocorticoid involvement in memory formation in a rat model for traumatic memory. Stress. 2002; 5(1):73-9.

Dal-Zotto S, Martí O, Armario A. Glucocorticoids are involved in the long-term effects of a single immobilization stress on the hypothalamic-pituitary-adrenal axis. Psychoneuroendocrinology. 2003; 28(8):992-1009.

Davis M. The role of the amygdala in fear and anxiety. Annu Rev Neurosci. 1992;15:353-75.

Davis M. The role of the amygdala in fear and anxiety. Annu Rev Neurosci. 1992; 15:353-75.

de Kloet ER, Joëls M, Holsboer F. Stress and the brain: from adaptation to disease. Nat Rev Neurosci. 2005; 6(6):463-75.

de Kloet ER, Oitzl MS, Joëls M. Stress and cognition: are corticosteroids good or bad guys? Trends Neurosci. 1999; 22(10):422-6.

de Quervain D, Schwabe L, Roozendaal B. Stress, glucocorticoids and memory: implications for treating fear-related disorders. Nat Rev Neurosci. 2017; 18(1):7-19.

de Quervain DJ, Roozendaal B, McGaugh JL. Stress and glucocorticoids impair retrieval of long-term spatial memory. Nature. 1998; 394(6695):787-90. 
Drevets WC, Videen TO, Price JL, Preskorn SH, Carmichael ST, Raichle ME. A functional anatomical study of unipolar depression. J Neurosci. 1992; 12(9): 3628-41.

Duque EA, Munhoz CD. The Pro-inflammatory Effects of Glucocorticoids in the Brain. Front Endocrinol. 2016; 7: 78.

Duvarci S, Pare D. Glucocorticoids enhance the excitability of principal basolateral amygdala neurons. J Neurosc.i. 2007; 27:4482-4491.

Falls WA, Miserendino MJ, Davis M. Extinction of fear-potentiated startle: blockade by infusion of an NMDA antagonist into the amygdala. J Neurosci. 1992; 12(3):854-63.

Fanselow MS, Bolles RC. Naloxone and shock-elicited freezing in the rat. J Comp Physiol Psychol. 1979; 93(4):736-44.

Farrell MR, Sayed JA, Underwood AR, Wellman CL. Lesion of infralimbic cortex occludes stress effects on retrieval of extinction but not fear conditioning. Neurobiol Learn Mem. 2010; 94: 240-246.

Fendt M, Fanselow MS. The neuroanatomical and neurochemical basis of conditioned fear. Neurosci Biobehav Rev. 1999; 23(5):743-60.

Foa EB, Zinbarg R, Rothbaum BO. Uncontrollability and unpredictability in posttraumatic stress disorder: an animal model. Psychol Bull. 1992; 112:218-38.

Fox C, Merali Z, Harrison C. Therapeutic and protective effect of environmental enrichment against psychogenic and neurogenic stress. Behav Brain Res. 2006; 75:18.

Francis DD, Diorio J, Plotsky PM, Meaney MJ. Environmental enrichment reverses the effects of maternal separation on stress reactivity. J Neurosci. 2002; 22:7840-7843.

Friske JE, Gammie SC. Environmental enrichment alters plus maze, but not maternal defense performance in mice. Physiol Behav. 2005; 85(2):187-94.

Gallagher M, Holland PC. The amygdala complex: multiple roles in associative learning and attention. Proc Natl Acad Sci U S A. 1994; 91(25):11771-6.

Ganon-Elazar E, Akirav I. Cannabinoid receptor activation in the basolateral amygdala blocks the effects of stress on the conditioning and extinction of inhibitory avoidance. J Neurosci. 2009; 29: 11078-11088. 
Ganon-Elazar E, Akirav I. Cannabinoid receptor activation in the basolateral amygdala blocks the effects of stress on the conditioning and extinction of inhibitory avoidance. J Neurosci. 2009; 29: 11078-11088.

Gore F, Schwartz EC, Brangers BC, Aladi S, Stujenske JM, Likhtik E, Russo MJ, Gordon JA, Salzman CD, Axel R. Neural Representations of Unconditioned Stimuli in Basolateral Amygdala Mediate Innate and Learned Responses. Cell. 2015; 162(1):134-45.

Grillo CA, Risher M, Macht VA, Bumgardner AL, Hang A, Gabriel C et al. Repeated restraint stress-induced atrophy of glutamatergic pyramidal neurons and decreases in glutamatergic efflux in the rat amygdala are prevented by the antidepressant agomelatine. Neuroscience. 2015; 284: 430-443.

Gross C, Hen R. The developmental origins of anxiety. Nat Rev Neurosci. 2004; 5:545552.

Hale MW, Bouwknecht JA, Spiga F, Shekhar A, Lowry CA. Exposure to high- and lowlight conditions in an open-field test of anxiety increases c-Fos expression in specific subdivisions of the rat basolateral amygdaloid complex. Brain Res Bull. 2006; 71: 174182.

Hatfield T1, Han JS, Conley M, Gallagher M, Holland P. Neurotoxic lesions of basolateral, but not central, amygdala interfere with Pavlovian second-order conditioning and reinforcer devaluation effects. J Neurosci. 1996; 16(16):5256-65.

Haubensak W, Kunwar PS, Cai H, Ciocchi S, Wall NR, Ponnusamy R, Biag J, Dong HW, Deisseroth K, Callaway EM, Fanselow MS, Lüthi A, Anderson DJ. Genetic dissection of an amygdala microcircuit that gates conditioned fear. Nature. 2010; 468(7321):270-6.

Herry C, Ciocchi S, Senn V, Demmou L, Müller C, Lüthi A. Switching on and off fear by distinct neuronal circuits. Nature. 2008; 454(7204):600-6.

Herry C, Ferraguti F, Singewald N, Letzkus JJ, Ehrlich I, Lüthi A. Neuronal circuits of fear extinction. Eur J Neurosci. 2010; 31(4):599-612.

Hobin JA, Goosens KA, Maren S. Context-dependent neuronal activity in the lateral amygdala represents fear memories after extinction. J Neurosci. 2003; 23(23):8410-6. 
Hoffman AN, Lorson NG, Sanabria F, Foster Olive M, Conrad CD. Chronicstress disrupts fear extinction and enhances amygdala and hippocampal Fos expression in an animal model of post-traumatic stress disorder. Neurobiol Learn Mem. 2014; 112: 139-147.

Holmes A, Wellman CL. Stress-induced prefrontal reorganization and executive dysfunction in rodents. Neurosci Biobehav Rev. 2009; 33: 773-783.

Holmes NM, Parkes SL, Killcross AS, Westbrook RF. The basolateral amygdala is critical for learning about neutral stimuli in the presence of danger, and the perirhinal cortex is critical in the absence of danger. J Neurosci. 2013; 33(32):13112-25.

Hull AM. Neuroimaging findings in post-traumatic stress disorder. Systematic review. Br J Psychiatry. 2002; 181:102-110.

Huzard D, Mumby DG, Sandi C, Poirier GL, van der Kooij MA. The effects of extrinsic stress on somatic markers and behavior are dependent on animal housing conditions. Physiol Behav. 2015; 151:238-45.

Izquierdo A, Wellman CL, Holmes A. Brief uncontrollable stress causes dendritic retraction in infralimbic cortex and resistance to fear extinction in mice. J Neurosci. 2006; 6: 5733-5738.

Janak PH, Tye KM. From circuits to behaviour in the amygdala. Nature. 2015; 517(7534):284-92.

Jimenez SA, Maren S. Nuclear disconnection within the amygdala reveals a direct pathway to fear. Learn Mem. 2009; 16(12):766-8.

Joëls M, Pu Z, Wiegert O, Oitzl MS, Krugers HJ. Learning under stress: how does it work? Trends Cogn Sci. 2006; 10(4):152-8.

Johansen JP, Hamanaka H, Monfils MH, Behnia R, Deisseroth K, Blair HT, LeDoux JE. Optical activation of lateral amygdala pyramidal cells instructs associative fear learning. Proc Natl Acad Sci U S A. 2010; 107(28):12692-7.

KadmielM, Cidlowski A. Glucocorticoid receptor signaling in health and disease. Trends Pharmacol Sci. 2013; 34(9): 518-530. 
Kaufer D, Ogle WO, Pincus ZS, Clark KL, Nicholas AC, Dinkel KM, et al. Restructuring the neuronal stress response with anti-glucocorticoid gene delivery. Nat Neurosci. $2004 ; 7(9): 947-53$.

Kavushansky A, Richter-Levin G. Effects of stress and corticosterone on activity and plasticity in the amygdala. J Neurosci Res. 2006; 84:1580-1587.

Kellett J, Kokkinidis L. Extinction deficit and fear reinstatement after electrical stimulation of the amygdala: implications for kindling-associated fear and anxiety. Neuroscience. 2001; 127(2):277-87.

Kim H, Yi JH, Choi K, Hong S, Shin KS, Kang SJ. Regional differences in acute corticosterone-induced dendritic remodeling in the rat brain and their behavioral consequences. BMC Neuroscience. 2014; 15:65.

Kim J, Pignatelli M, Xu S, Itohara S, Tonegawa S. Antagonistic negative and positive neurons of the basolateral amygdala. Nat Neurosci. 2016; 19(12):1636-1646.

Kirby ED, Friedman AR, Covarrubias D, Ying C, Sun WG, Goosens KA, Sapolsky RM, Kaufer D. Basolateral amygdala regulation of adult hippocampal neurogenesis and fear-related activation of newborn neurons. Mol Psychiatry. 2012; 17(5):527-36.

Kluge M, Schüssler P, Künzel HE, Dresler M, Yassouridis A, Steiger A. Increased nocturnal secretion of ACTH and cortisol in obsessive compulsive disorder. J Psychiatr Res. 2007; 41(11):928-33.

Knapska E, Radwanska K, Werka T, Kaczmarek L. Functional internal complexity of amygdala: focus on gene activity mapping after behavioral training and drugs of abuse. Physiol. Rev. 2007; 87, 1113-1173.

Knox D, George SA, Fitzpatrick CJ, Rabinak CA, Maren S, Liberzon I. Single prolonged stress disrupts retention of extinguished fear in rats. Learn Mem. 2012a; 19:43-9.

Knox D, Nault T, Henderson C, Liberzon I. Glucocorticoid receptors and extinc-tion retention deficits in the single prolonged stress model. Neuroscience. 2012b; 223:16373.

Koe AS, Ashokan A, Mitra, R. Short environmental enrichment in adulthood reverses anxiety and basolateral amygdala hypertrophy induced by maternal separation. Transl. Psychiatry. 2016; 6, 1-7. 
Kuhlmann S, Wolf OT. Arousal and cortisol interact in modulating memory consolidation in healthy young men. Behav Neurosci. 2006; 120(1):217-23.

LaBar KS, Gatenby JC, Gore JC, LeDoux JE, Phelps EA. Human amygdala activation during conditioned fear acquisition and extinction: a mixed-trial fMRI study. Neuron. 1998; 20(5):937-45.

Laemmli UK. Cleavage of structural proteins during the assembly of the head of bacteriophage T4. Nature. 1970; 15;227(5259):680-5.

Laurent V, Marchand AR, Westbrook RF. The basolateral amygdala is necessary for learning but not relearning extinction of context conditioned fear. Learn Mem. 2008; 15(5):304-14.

Laurent V, Westbrook RF. Distinct contributions of the basolateral amygdala and the medial prefrontal cortex to learning and relearning extinction of context conditioned fear. Learn Mem. 2008; 15(9):657-66.

Laurent V, Westbrook RF. Inactivation of the infralimbic but not the prelimbic cortex impairs consolidation and retrieval of fear extinction. Learn Mem. 2009; 25;16(9):5209.

Laurent V, Westbrook RF. Role of the basolateral amygdala in the reinstatement and extinction of fear responses to a previously extinguished conditioned stimulus. Learn Mem. 2010; 17(2):86-96.

Ledgerwood L, Richardson R, Cranney J. Effects of D-cycloserine on extinction of conditioned freezing. Behav Neurosci. 2003; 117(2):341-9.

LeDoux JE. Emotion circuits in the brain. Annu Rev Neurosci. 2000; 23:155-84.

LeDoux JE. The emotional brain, fear, and the amygdala. Cell Mol Neurobiol. 2003; 23(4-5):727-38.

Lee $\mathrm{H} 1$, Kim JJ. Amygdalar NMDA receptors are critical for new fear learning in previously fear-conditioned rats. J Neurosci. 1998; 18(20):8444-54.

Livneh U, Paz R. Aversive-bias and stage-selectivity in neurons of the primate amygdala during acquisition, extinction, and overnight retention. J. Neurosci. 2012; 32, 8598-8610. 
Long VA, Fanselow MS. Stress-enhanced fear learning in rats is resistant to the effects of immediate massed extinction. Stress. 2012; 15: 627-636.

Maren S, Chang CH. Recent fear is resistant to extinction. Proc Natl Acad Sci. 2006; 21: 18020-18025.

Maren S, Phan KL, Liberzon I. The contextual brain: implications for fear conditioning, extinction and psychopathology. Nat Rev Neurosci. 2013; 14(6):417-28.

Maren S, Quirk GJ. Neuronal signalling of fear memory. Nat Rev Neurosci. 2004; 5:844-52.

Maren S, Yap SA, Goosens KA. The amygdala is essential for the development of neuronal plasticity in the medial geniculate nucleus during auditory fear conditioning in rats. J Neurosci. 2001; 21(6):RC135.

Maroun M, Ioannides PJ, Bergman KL, Kavushansky A, Holmes A, Wellman CL. Fear extinction deficits following acute stress associate with increased spine density and dendritic retraction in basolateral amygdala neurons. Eur J Neurosci. 2013; 38: $2611-$ 2620.

McDonald AJ. Cortical pathways to the mammalian amygdala. Prog Neurobiol. 1998; 55(3):257-332.

McEwen BS, Eiland L, Hunter RG, Miller MM. Stress and anxiety: structural plasticity and epigenetic regulation as a consequence of stress. Neuropharmacology. 2012; 62(1):3-12.

McEwen BS, Gray JD, Nasca C. 60 YEARS OF NEUROENDOCRINOLOGY: Redefining neuroendocrinology: stress, sex and cognitive and emotional regulation. $J$ Endocrinol. 2015; 226(2):T67-83.

McEwen BS, Sapolsky RM. Stress and cognitive function. Curr Opin Neurobiol. 1995; 5(2):205-16.

McEwen BS. Plasticity of the hippocampus: adaptation to chronic stress and allostatic load. Ann N Y Acad Sci. 2001; 933:265-77.

McFarlane AC. The long-term costs of traumatic stress: intertwined physical and psychological consequences. World Psychiatry. 2010; 9:3-10. 
McGaugh JL, Roozendaal B. Role of adrenal stress hormones in forming lasting memories in the brain. Curr Opin Neurobiol. 2002; 12(2):205-10.

McGaugh JL. The amygdala modulates the consolidation of memories of emotionally arousing experiences. Annu Rev Neurosci. 2004; 27:1-28.

McIntyre CK, McGaugh JL, Williams CL. Interacting brain systems modulate memory consolidation. Neurosci Biobehav Rev. 2012; 36(7):1750-62.

Meyer RM, Burgos-Robles A, Liu E, Correia SS, Goosens KA. A ghrelin-growth hormone axis drives stress-induced vulnerability to enhanced fear. Mol Psychiatry. 2014; 19(12): 1284-1294.

Milad MR, Quirk GJ. Fear extinction as a model for translational neuroscience: ten years of progress. Annu Rev Psychol. 2012; 63:129-51.

Mitra R, Ferguson D, Sapolsky RM. SK2 potassium channel over-expression in basolateral amygdala reduces anxiety, stress-induced corticosterone and dendritic arborization. Mol. Psychiatry. 2009; 14 (9): 847-827.

Mitra R, Jadhav S, McEwen BS, Vyas A, Chattarji S. Stress duration modulates the spatiotemporal patterns of spine formation in the basolateral amygdala. Proceedings of the National Academy of Sciences. 2005; 102:9371-9376.

Mitra R, Sapolsky RM. Expression of chimeric estrogen-glucocorticoid-receptor in the amygdala reduces anxiety. Brain Research. 2010; 1342:33-38.

Mitra R, Sapolsky RM. Acute corticosterone treatment is sufficient to induce anxiety and amygdaloid dendritic hypertrophy. Proc Natl Acad Sci U S A. 2008; 105:55735578.

Mohammed AH, Zhu SW, Darmopil S, Hjerling-Leffler J, Ernfors P, Winblad B, Diamond MC, Eriksson PS, Bogdanovic N. Environmental enrichment and the brain. Progress in brain research. 2002; 138:109-133.

Moncek F, Duncko R, Johansson BB, Jezova D. Effect of environmental enrichment on stress related systems in rats. J Neuroendocrinol. 2004; 16(5):423-31.

Morgan M, LeDoux J. Differential contribution of dorsal and ventral medial prefrontal cortex to the acquisition and extinction of conditioned fear in rats. Behav Neurosci. 1995; 109: 681-688. 
Morley-Fletcher S, Rea M, Maccari S, Laviola G. Environmental enrichment during adolescence reverses the effects of prenatal stress on play behaviour and HPA axis reactivity in rats. Eur J Neurosci. 2003; 18(12):3367-74.

Muigg, P, Hetzenauer, A, Hauer, G, Huschild, M, Gaburro, S, \& Frank, E. Impaired extinction of learned fear in rats selectively bred for high anxiety- Evidence of altered neuronal processing in prefrontal-amygdala pathways. Eur J Neurosci. 2008; 28, 2299-2309.

Muramoto K, Ono T, Nishijo H, Fukuda M. Rat amygdaloid neuron responses during auditory discrimination. Neuroscience. 1993; 52, 621-636.

Myers KM, Davis M. Behavioral and neural analysis of extinction. Neuron. 2002; 36(4):567-84.

Namburi P, Beyeler A, Yorozu S, Calhoon GG, Halbert SA, Wichmann R, Holden SS, Mertens KL, Anahtar M, Felix-Ortiz AC, Wickersham IR, Gray JM, Tye KM. A circuit mechanism for differentiating positive and negative associations. Nature. 2015; 520(7549):675-8.

Nieminen SA, Sirvio J, Teittinen K, Pitkanen A, Airaksinen MM, Riekkinen P. Amygdala kindling increased fear-response, but did not impair spatial memory in rats. Physiology \& behavior. 1992; 51:845-849.

Novaes LS, Dos Santos NB, Batalhote RF, Malta MB, Camarini R, Scavone C, Munhoz CD. Environmental enrichment protects against stress-induced anxiety: Role of glucocorticoid receptor, ERK, and CREB signaling in the basolateral amygdala. Neuropharmacology. 2017; 113:457-466.

Okuda S, Roozendaal B, McGaugh JL. Glucocorticoid effects on object recognition memory require training-associated emotional arousal. Proc Natl Acad Sci U S A. $2004 ; 101(3): 853-8$.

Orr SP, Metzger LI, Pitman RK. Psychophysiology of post-traumatic stress disorder Psychiatr Clin North Am 2002; 25:279-935(2):271-93.

Otto C1, Reichardt HM, Schütz G. Absence of glucocorticoid receptor-beta in mice. J Biol Chem. 1997; 272(42):26665-8. 
Padival MA, Blume SR, Rosenkranz JA. Repeated restraint stress exerts different impact on structure of neurons in the lateral and basal nuclei of the amygdala. Neuroscience. 2013; 246: 230-242.

Patel PD, Lopez JF, Lyons DM, Burke S, Wallace M, Schatzberg AF. Glucocorticoid and mineralocorticoid receptor mRNA expression in squirrel monkey brain. Journal of Psychiatric Research. 2000; 34:383-392.

Paton JJ, Belova MA, Morrison SE, Salzman CD. The primate amygdala represents the positive and negative value of visual stimuli during learning. Nature. 2006; 439, 865-870.

Pelletier JG, Likhtik E, Filali M, Paré D. Lasting increases in basolateral amygdala activity after emotional arousal: implications for facilitated consolidation of emotional memories. 2005; 12(2):96-102.

Perusini JN, Meyer EM, Long VA, Rau V, Nocera N, Avershal J, Maksymetz J, Spigelman I, Fanselow MS. Induction and Expression of Fear Sensitization Caused by Acute Traumatic Stress. Neuropsychopharmacology. 2016; 41(1):45-57.

Pessoa L, McKenna M, Gutierrez E, Ungerleider LG. Neural processing of emotional faces requires attention. Proc Natl Acad Sci U S A. 2002; 99(17):11458-63.

Phelps EA, LeDoux JE. Contributions of the amygdala to emotion processing: from animal models to human behavior. Neuron. 2005; 48(2):175-87.

Pietropaolo S, Feldon J, Yee, BK. Environmental enrichment eliminates the anxiety phenotypes in a triple transgenic mouse model of Alzheimer's disease. Cogn Affect Behav Neurosci. 2014; 14:996-1008.

Pitman RK, Rasmusson AM, Koenen KC, Shin LM, Orr SP, Gilbertson MW, Milad MR, Liberzon I. Biological studies of post-traumatic stress disorder. Nat Rev Neurosci. 2012; 13(11):769-87.

Pryce, CR. Postnatal ontogeny of expression of the corticosteroid receptor genes in mammalian brains: Inter-species and intra-species differences. Brain Research Reviews. 2008; 57:596-605.

Quirk GJ, Mueller D. Neural mechanisms of extinction learning and retrieval. Neuropsychopharmacology. 2008; 33(1):56-72. 
Quirk GJ, Repa C, LeDoux JE. Fear conditioning enhances short-latency auditory responses of lateral amygdala neurons: parallel recordings in the freely behaving rat. Neuron. 1995; 15:1029-39.

Quirk GJ, Russo GK, Barron JL, Lebron K (2000). The role of ventromedial prefrontal cortex in the recovery of extinguished fear. J Neurosci. 2000; 20: 6225-6231.

Radley JJ, Rocher AB, Rodriguez A, Ehlenberger DB, Dammann M, McEwen BS, Morrison JH, Wearne SL, Hof PR. Repeated stress alters dendritic spine morphology in the rat medial prefrontal cortex. J Comp Neurol. 2008; 507(1):1141-50.

Rao RP, Anilkumar S, McEwen B, Chattarji S. Glucocorticoids protect against the delayed behavior and cellular effects of acute stress on the amygdala. Biol. Psychiatry. 2012; 72 (6), 466-475.

Ratman R, Berghe WV, Dejager L, Libert C, Tavernier J, Beck IM, de Bosscher K. How glucocorticoid receptors modulate the activity of other transcription factors: a scope beyond tethering. Mol Cell Endocrinol. 2013; 380(1-2):41-54.

Rauch SL, Shin LM, Phelps EA. Neurocircuitry models of posttraumatic stress disorder and extinction: Human neuroimaging research:past, present, and future. Biol. Psychiatry. 2006; 60:376-382.

Ravenelle R, Santolucito HB, Byrnes EM, Byrnes JJ, Donaldson ST. Housing environment modulates physiological and behavioral responses toanxiogenic stimuli in trait anxiety male rats. Neuroscience. 2014; 270, 76-87.

Repa JC, Muller J, Apergis J, Desrochers TM, Zhou Y, LeDoux JE. Two different lateral amygdala cell populations contribute to the initiation and storage of memory. Nat Neurosci. 2001; 4(7):724-31.

Ressler KJ, Nemeroff CB. Role of norepinephrine in the pathophysiology and treatment of mood disorders. Biol Psychiatry. 1999; 46(9):1219-33.

Rodrigues SM, LeDoux JE, Sapolsky RM.The influence of stress hormones on fear circuitry. Annual review of neuroscience. 2009; 32:289-313.

Rodriguez Manzanares PA, Isoardi NA, Carrer HF, Molina VA. Previous stress facilitates fear memory, attenuates GABAergic inhibition, and increases synaptic plasticity in the rat basolateral amygdala. J Neurosci. 2005; 25:8725-8734. 
Romanski LM, Clugnet MC, Bordi F, LeDoux JE. Somatosensory and auditory convergence in the lateral nucleus of the amygdala. Behav. Neurosci. 1993; 107, 444450.

Roozendaal B, McGaugh JL. Amygdaloid nuclei lesions differentially affect glucocorticoid-induced memory enhancement in an inhibitory avoidance task. Neurobiol Learn Mem. 1996; 65(1):1-8.

Roozendaal B, Williams CL, McGaugh JL. Glucocorticoid receptor activation in the rat nucleus of the solitary tract facilitates memory consolidation: involvement of the basolateral amygdala. Eur J Neurosci. 1999; 11(4):1317-23.

Roozendaal B, Williams CL, McGaugh JL. Glucocorticoid receptor activation in the rat nucleus of the solitary tract facilitates memory consolidation: involvement of the basolateral amygdala. Eur J Neurosci. 1999;11(4):1317-23.

Rosen JB, Schulkin J. From normal fear to pathological anxiety. Psychological review. 1998; 105:325-350.

Rothbaum BO, Davis M. Applying learning principles to the treatment of post-trauma reactions. Ann N Y Acad Sci. 2003; 1008:112-21.

Roy V, Belzung C, Delarue C, Chapillon P. Environmental enrichment inBALB/c mice: effects in classical tests of anxiety and exposure to a predatoryodor. Physiol. Behav. $2001 ; 74,313-320$.

Russo SJ, Wilkinson MB, Mazei-Robison MS, Dietz DM, Maze I, Krishnan V, Renthal W, Graham A, Birnbaum SG, Green TA, Robison B, Lesselyong A, Perrotti LI, Bolaños CA, Kumar A, Clark MS, Neumaier JF, Neve RL, Bhakar AL, Barker PA, Nestler EJ. Nuclear factor kappa B signaling regulates neuronal morphology and cocaine reward. J Neurosci. 2009;18;29(11):3529-37.

Sah P, Faber ES, Lopez De Armentia M, Power J. The amygdaloid complex: anatomy and physiology. Physiol Rev. 2003; 83(3):803-34.

Salzman CD, Fusi S. Emotion, cognition, and mental state representation in amygdala and prefrontal cortex. Annu Rev Neurosci. 2010; 33:173-202.

Santini E, Quirk GJ, Porter JT. Fear conditioning and extinction differentially modify the intrinsic excitability of infralimbic neurons. J Neurosci. 2008; 28(15):4028-36. 
Sapolsky RM. Glucocorticoids and hippocampal atrophy in neuropsychiatric disorders. Arch Gen Psychiatry. 2000; 57(10):925-35.

Sarter M, Markowitsch HJ. Involvement of the amygdala in learning and memory: a critical review, with emphasis on anatomical relations. Behav Neurosci. 1985; 99(2):342-80.

Shin, LM, Wright, Cl, Cannistraro, PA, Wedig, MM, McMullin, K, Martis, B, Macklin, ML, Lasko, NB, Cavanagh, SR, Krangel, TS, Orr, SP, Pitman, R, Whalen, k, Rauch, PJSL. A functional magnetic resonance imaging study of amygdala and medial prefrontal cortex responses to overtly presented fearful faces in posttraumatic stress disorder. Arch. Gen. Psychiatry. 2005; 62, 273-281.

Shumake J, Barrett D, Gonzalez-Lima F. Behavioral characteristics of rats predisposed to learned helplessness: Reduced reward sensitivity, increased novelty seeking, and persistent fear memories. Behav Brain Res. 2005; 164: 222-230.

Sierra-Mercado D, Padilla-Coreano N, Quirk GJ. Dissociable roles of prelimbic and infralimbic cortices, ventral hippocampus, and basolateral amygdala in the expression and extinction of conditioned fear. Neuropsychopharmacology. 2011;36(2):529-38.

Silveira MC, Sandner G, Graeff FG. Induction of Fos immunoreactivity in the brain by exposure to the elevated plus-maze. Behav Brain Res. 1993; 56: 115-118.

Simmons AN, Matthews SC, Strigo IA, Baker DG, Donovan HK, Motezadi A, Stein MB, Paulus MP. Altered amygdala activation during face processing in Iraqi and Afghanistani war veterans. Biol Mood Anxiety Disord. 2011; 12;1(1):6.

Singewald N, Schmuckermair C, Whittle N, Holmes A, Ressler KJ. Pharmacology of cognitive enhancers for exposure-based therapy of fear, anxiety and trauma-related disorders. Pharmacol Ther. 2015; 149:150-90.

Soravia LM, Heinrichs M, Aerni A, Maroni C, Schelling G, Ehlert U, Roozendaal B, de Quervain DJ. Glucocorticoids reduce phobic fear in humans. Proc Natl Acad Sci U S A. 2006; 103(14):5585-90.

Stuber GD, Sparta DR, Stamatakis AM, van Leeuwen WA, Hardjoprajitno JE, Cho S, Tye KM, Kempadoo KA, Zhang F, Deisseroth K, Bonci A. Excitatory transmission from the amygdala to nucleus accumbens facilitates reward seeking. Nature. 2011; 475(7356):377-80. 
Thompson BM, Baratta MV, Biedenkapp JC, Rudy JW, Watkins LR, Maier SF. Activation of the infralimbic cortex in a fear context enhances extinction learning. Learn Mem. 2010; 17(11):591-9.

Toledo-Rodriguez M, Pitiot A, Paus T, Sandi C. Stress during puberty boosts metabolic activation associated with fear-extinction learning in hippocampus, basal amygdala and cingulate cortex. Neurobiol Learn Mem. 2012; 98: 93-101.

Vallès A, Martí O, Armario A. Long-term effects of a single exposure to immobilization stress on the hypothalamic-pituitary-adrenal axis: transcriptional evidence for a progressive desensitization process. Eur J Neurosci. 2003; 18(6):1353-61.

van Praag H, Kempermann G, Gage FH. Neural consequences of environmental enrichment. Nat Rev Neurosci 2000;1:191-198.

Vuilleumier P, Armony JL, Driver J, Dolan RJ. Effects of attention and emotion on face processing in the human brain: an event-related fMRI study. Neuron. 2001; 30(3):82941.

Vyas A, Jadhav S, Chattarji S. Prolonged behavioral stress enhances synaptic connectivity in the basolateral amygdala. Neuroscience. 2006; 143: 387-393.

Vyas A, Mitra R, Shankaranarayana RBS, Chattarji S. Chronic stress induces contrasting patterns of dendritic remodeling in hippocampal and amygdaloid neurons. J Neurosci. 2002; 22:6810-6818.

Walker DL, Ressler KJ, Lu KT, Davis M. Facilitation of conditioned fear extinction by systemic administration or intra-amygdala infusions of D-cycloserine as assessed with fear-potentiated startle in rats. J Neurosci. 2002; 22(6):2343-51.

Wang DV, Wang F, Liu J, Zhang L, Wang Z, Lin L. Neurons in the amygdala with response-selectivity for anxiety in two ethologically based tests. PLoS One. 2011; 6(4):e18739.

Welberg L, Thrivikraman KV, Plotsky PM. Combined pre- and postnatal environmental enrichment programs the HPA axis differentially in male and female rats. Psychoneuroendocrinology. 2006; 31(5):553-64.

Wilber AA, Walker AG, Southwood CJ, Farrell MR, Lin GL, Rebec GV et al. Chronic stress alters neural activity in medial prefrontal cortex during retrieval of extinction. Neuroscience. 2011; 174: 115-131. 
Wolff SB, Grundemann J, Tovote P, Krabbe S, Jacobson GA, Muller C, Herry C, Ehrlich I, Friedrich RW, Letzkus JJ, Luthi A. Amygdala interneuron subtypes control fear learning through disinhibition. Nature. 2014; 509, 453-458.

Xu L, Anwyl R, Rowan MJ. Behavioural stress facilitates the induction of long-term depression in the hippocampus. Nature. 1997; 387(6632):497-500.

Xu L, Anwyl R, Rowan MJ. Spatial exploration induces a persistent reversal of longterm potentiation in rat hippocampus. Nature. 1998; 394(6696):891-4.

Yamamoto S, Morinobu S, Fuchikami M, Kurata A, Kozuru T, Yamawaki S. Effects of single prolonged stress and D-cycloserine on contextual fear extinction and hippocampal NMDA receptor expression in a rat model of PTSD. Neuropsychopharmacology. 2008; 33: 2108-2116.

Yamamoto S, Morinobu S, Fuchikami M, Kurata A, Kozuru T, Yamawaki S. Effects of single prolonged stress and D-cycloserine on contextual fear extinction and hippocampal NMDA receptor expression in a rat model of PTSD. Neuropsychopharmacology. 2008; 33: 2108-2116.

Yang YL, Chao PK, Lu KT. Systemic and intra-amygdala administration of glucocorticoid agonist and antagonist modulate extinction of conditioned fear. Neuropsychopharmacology. 2006; 31(5):912-24.

Yehuda R, LeDoux J. Response variation following trauma: a translational neuroscience approach to understanding PTSD. Neuron. 2007; 56(1):19-32.

Yehuda R. Advances in understanding neuroendocrine alterations in PTSD and their therapeutic implications. Ann N Y Acad Sci. 2006; 1071:137-66.

Yehuda R. Biology of posttraumatic stress disorder. J Clin Psychiatry. 2001; 62(17):416.

Yehuda R. Post-traumatic stress disorder. N Engl J Med. 2002; 346:108-14.

Yiu AP, Mercaldo V, Yan C, Richards B, Rashid AJ, Hsiang HL, Pressey J, Mahadevan V, Tran MM, Kushner SA, Woodin MA, Frankland PW, Josselyn SA. Neurons are recruited to a memory trace based on relative neuronal excitability immediately before training. Neuron. 2014; 83(3):722-35. 
Zoladz PR, Clark B, Warnecke A, Smith L, Tabar J, Talbot JN. Pre-learning stress differentially affects long-term memory for emotional words, depending on temporal proximity to the learning experience. Physiol Behav. 2011; 103(5):467-76. 